\title{
Administration of Rhodiola kirilowii Extracts during Mouse Pregnancy and Lactation Stimulates Innate but Not Adaptive Immunity of the Offspring
}

\author{
Sławomir Lewicki, ${ }^{1}$ Ewa Skopińska-Różewska, ${ }^{1,2}$ Aleksandra Brewczyńska, ${ }^{1}$ and \\ Robert Zdanowski ${ }^{1}$ \\ ${ }^{1}$ Department of Regenerative Medicine and Cell Biology, Military Institute of Hygiene and Epidemiology, Kozielska 4, 01-163 \\ Warsaw, Poland \\ ${ }^{2}$ Pathomorphology Department, Center for Biostructure Research, Warsaw Medical University, Chałubińskiego 5, \\ 02-004 Warsaw, Poland
}

Correspondence should be addressed to Robert Zdanowski; rztox@yahoo.com

Received 2 March 2017; Accepted 14 September 2017; Published 30 October 2017

Academic Editor: Daniel Ortuño-Sahagún

Copyright (C) 2017 Sławomir Lewicki et al. This is an open access article distributed under the Creative Commons Attribution License, which permits unrestricted use, distribution, and reproduction in any medium, provided the original work is properly cited.

\begin{abstract}
The use of antibiotics during pregnancy and lactation is associated with an increased risk of developmental disorders. One of the natural medicinal plants-Rhodiola kirilowii, widely used as an immunostimulant in adults-might be a good alternative to antibiotic treatment. The aim of present study was to assess whether daily oral administration of $20 \mathrm{mg} / \mathrm{kg}$ of Rhodiola kirilowii aqueous (RKW) or 50\% hydroalcoholic (RKW-A) extracts affected hematological and immunological parameters of 6-week-old mouse progeny. There was no significant change in hematological parameters of blood with the exception of hemoglobin, which was significantly higher (about 4\%) in RKW group. Offspring of mothers fed Rhodiola kirilowii extracts had increased percentage of granulocytes and decreased percentage of lymphocytes. These changes correlated with decreased percentage of $\mathrm{CD}^{+} / \mathrm{CD}^{+}$T-cells (RKW and RKW-A), decrease of $\mathrm{CD}^{+}$cells, and increase percentage of NK cells in RKW group. In addition, both types of Rhodiola kirilowii extracts stimulated granulocyte phagocytosis and increased level of respiratory burst. In conclusion, the long-term supplementation of mouse mothers during pregnancy and lactation with RKW or RKW-A extracts affects the immune system of their progeny. These results should be taken into consideration before administration of Rhodiola kirilowii to pregnant and lactating women.
\end{abstract}

\section{Introduction}

Treatment of illnesses during pregnancy and in early infancy can be problematic because of its potential effects on development of infant immune system [1]. Vast majority of infections are treated with antibiotics that cause plethora of side effects. FDA (Food and Drug Administration) of the U.S. Department of Health and Human Services created a list of antibiotics, divided into 5 groups (A, B, C, D, and X) depending on the potential risk of side effects. Only antibiotics from group A are considered as being safe to use during pregnancy and lactation [2]. The validity of such a classification was confirmed in studies conducted on mouse model. Using experimental model of pregnant mice,
Skopińska-Różewska and collaborators showed that prenatal exposure to antibiotics modifies the immune system in progeny $[3,4]$. Moreover, the offspring of mothers treated during pregnancy with antibiotics from penicillin and cephalosporin group (group B) showed an altered reactivity of the immune system manifested by decreased intensity of cellular response and increased intensity of humoral immune response to pathogens. In addition, a prenatal and postnatal exposure to antibiotics is associated with an increased risk of asthma and obesity in childhood [5].

The overuse of antibiotics not only in medicine but also in food industry causes increasing antibiotic resistance and other side effects [6-8]. Thus, there is an increasing demand for a natural antimicrobial and immunostimulatory 
medications, which could be safely administered during pregnancy; the herbs from the Rhodiola genus seem to be an excellent candidate.

Rhodiola genus from Crassulaceae family consists of more than 200 species out of which over 20 species have medicinal properties. They were used in traditional medicine of Asia and Europe as tonic, adaptogen, antidepressant, and anti-inflammatory drugs, and for these properties, they are being tested recently in some clinical trials [9-11]. Clinical trials showed lack of adverse effects and interactions between Rhodiola extracts and other drugs. Plants from Rhodiola species are known not only as a diet supplement that supports mental and physical performance of the body but also for the beneficial antitumor, antimicrobial, and immunomodulatory activity $[12,13]$. Importantly, Rhodiola has antiviral and antimicrobial properties against hepatitis $\mathrm{C}$ virus (HCV) and Mycobacterium tuberculosis [14, 15]. Currently, several novel clinical therapies based on the abilities of Rhodiola extracts to stimulate the immune system are under development [16].

All these properties make Rhodiola an attractive source for producing various medications. Biological activity of Rhodiola spp. depends on the phytochemicals such as polyphenols, phytosterols, caretonoids, saponines, and alkaloids. Using mouse model, we showed that Rhodiola kirilowii, $R$. rosea, and $R$. quadrifida extracts stimulated granulocyte and lymphocyte activity. Feeding mice with Rhodiola extracts for 7 days lowered intensity of Pseudomonas aeruginosa infection, increased blood leukocyte number, and had modulatory effect on their metabolic activity [17-22].

The aim of the present study was to investigate if and how $50 \%$ hydroalcoholic (RKW-A) and aqueous (RKW) extracts of underground (roots and rhizomes) parts of Rhodiola kirilowii administrated to pregnant and lactating mice stimulate innate immunity of the offspring.

\section{Material and Methods}

2.1. Plant Cultivation. Rhodiola kirilowii (Crassulaceae) plants were collected from the Institute of Natural Fibers and Medical Plants (Poznań, Poland) field cultivations of herbal plants. The taxonomic status of plant was confirmed with Flora of the Soviet Union (Vol. 9, 1939) and Flora of China (Vol. 8, 2001). A voucher specimen is kept in the herbarium of the Department of Botany, Breeding and Agriculture in Plewiska near Poznań.

2.2. Preparation of Extracts. Extracts have been prepared as previously described [23]. Briefly, for RKW extract production, the finely powdered $R$. kirilowii roots were extracted twice with water (first for $2 \mathrm{~h}$ and then for $1 \mathrm{~h}$ ) in a raw material to solvent ratio of $1: 5$, at temperature between 40 and $45^{\circ} \mathrm{C}$. Supernatants from each extraction were mixed together, centrifuged ( $15 \mathrm{~min}$, room temperature, $2000 \times \mathrm{g}$ ), and lyophilized. RKW extract contained about $14 \mu \mathrm{g}$ of analyzed polyphenols (kaempferol, epicatechin, quercetin, (+)-catechin, salidroside, fisetin, naringenin, luteoli, pcoumaric acid, ellagic acid, epigallocatechin, ferulic acid, chlorogenic acid, epicatechin gallate, and epigallocatechin gallate) per mg of dry extract. For RKW-A extract production, the finely powdered $R$. kirilowii roots were extracted with a $50 \%$ ethanol, in a raw material to solvent ratio of $1: 10$ using the percolation method. The percolates were lyophilized following distillation at $40-45^{\circ} \mathrm{C}$. RKW-A extract contained about $21 \mu \mathrm{g}$ of analyzed polyphenols.

Dry extract ratio (g of medical herb to $g$ final extract) was 5.09:1 for RKW and 3.27:1 for RKW-A. Extracts were stored at $-70^{\circ} \mathrm{C}$ until further use.

2.3. Animals. All animal experiments were conducted according to the Polish regulation and standards of the wellness of laboratory animals. All experiments were accepted by and conducted according to the ethical guidance of Local Bioethical Committee (permission 73/2011).

Experiments were performed on 6-week-old progeny of 70 adult inbred female BALB/c mice (8-9 weeks old; $~ 20 \mathrm{~g}$ weight; purchased from Mossakowski Medical Research Centre Polish Academy of Sciences, Warsaw, Poland), which were mated with adult males of the same strain. After confirmation of pregnancy (the presence of copulatory plug), $\mathrm{Balb} / \mathrm{c}$ females were fed daily (up to the 28th day after delivery) with lyophilized RKW or RKW-A extracts (20 mg of extract $/ \mathrm{kg}$ of body weight) dissolved in sterile water. The control group received sterile water only. To avoid stress related to gavage feeding and handling that can lead to miscarriage, 20 microliters of tested substance dissolved in sterile water was placed on one corn chip and served to the mouse in a petri dish. Female and male progenies were housed separately.

Mice were maintained under conventional conditions $\left(22.5-23^{\circ} \mathrm{C}\right.$, relative humidity $50-70 \%, 12 \mathrm{~h}$ day/night cycle) with free access to breeding rodent feed (Labofeed $\mathrm{H}$, Wytwórnia Pasz "Morawski") and water. Pups were withdrawn from mothers 24 days after delivery.

2.4. Blood Isolation. Mice were anesthetized (intraperitoneal injection of ketamine $(120 \mathrm{mg} / \mathrm{kg})$ and xylazine $(12 \mathrm{mg} / \mathrm{kg})$; Polypharm S.A., Warsaw, Poland), and retro-orbital blood was collected in the EDTA-containing tubes (for further hematological and immunological analysis) and heparincontaining tubes (for further phagocytosis and oxidative burst analysis).

2.5. Hematological Analysis. Blood ( $50 \mu \mathrm{l}$ from EDTA tubes) was analyzed in a hematological analyzer (Exigo veterinary hematological system; Boule Medical AB, Stockholm, Sweden). The following parameters were evaluated: WBC (white blood cells), RBC (red blood cells), HGB (hemoglobin), HCT (hematocrit), MCV (mean corpuscular volume), $\mathrm{MCH}$ (mean corpuscular hemoglobin), MCHC (mean corpuscular hemoglobin concentration), RDW\% (red cell distribution width (\%)), RDW-a (red cell distribution width absolute), PLT (platelets), and MPV (mean platelet volume). Additionally, WBC number and cell percentage from flow cytometry analysis were used to calculate a total number of lymphocytes, lymphocyte subpopulations, monocytes, and granulocytes per $\mu \mathrm{l}$ of blood. The results are presented as the mean \pm standard error. 
2.6. Determination of White Blood Cell Phenotype. Determination of WBC phenotype was performed as described previously for splenocyte phenotyping [24]. Briefly, the cells in whole blood ( $100 \mu \mathrm{l}$ from EDTA tubes) were labeled with the following fluorochrome-conjugated anti-mouse monoclonal antibodies: Mouse T lymphocyte Subset Antibody Cocktail with Isotype Control (hamster anti-mouse cluster of differentiation (CD) 3e, rat anti-mouse PE CD4, and rat anti-mouse CD8a; cat. number 558431), Mouse B Lymphocyte Activation Antibody Cocktail with Isotype Control (rat anti-mouse CD25, hamster anti-mouse CD69, and rat anti-mouse CD19), and rat anti-mouse CD335 (natural killer cell p46-related protein all purchased from BD Biosciences, Warsaw, Poland). Staining (20 min incubation at room temperature) was performed according to the manufacturer's instructions. Subsequently, the red blood cells were lysed (10 min, Lysing Solution 10x Concentrate; BD Biosciences) and remaining cells were washed twice with PBS. Phenotypic analysis was performed by flow cytometry (FACSCalibur; BD Biosciences). Additional phenotypic determination of WBC population (lymphocytes, monocytes, and granulocytes) was made using FSC/SSC parameters. The results are presented as the mean $\% \pm$ standard error of the mean of WBC (for lymphocytes, monocytes, and granulocytes) or lymphocytes (for CD3, CD4, CD8, CD19, and CD335 analysis) and also as mean \pm standard error of cell number in liter of blood.

2.7. Treg Analysis. Mouse Treg cell evaluation was performed analogously as previously described for Treg cells [25]. Briefly, cells in whole blood samples $(100 \mu \mathrm{l})$ were immunostained with $20 \mu \mathrm{l}$ of primary antibodies CD4-PerCP and CD25-APC (extracellular staining, BD Biosciences, Poland) or an appropriate isotype control for 20 minutes at room temperature. Subsequently, erythrocytes were lysed in BD FACS Lysing Solution for 10 minutes at room temperature. Remaining immune cells were fixed, permeabilized (fixation/permeabilization buffer) in accordance with the manufacturer's protocol (BD Pharmingen, Poland), and stained with $20 \mu \mathrm{l}$ of FoxP3 PE or isotype IgG1 kappa PE antibody ( $45 \mathrm{~min} / \mathrm{RT}$ in the dark). Afterwards, the cells were washed twice with PBS and fixed in $300 \mu \mathrm{l}$ of $1 \%$ PFA in PBS solution. The cells were counted by flow cytometry-10000 counts of CD4-PerCP-positive cells stopped the acquisition. Evaluation of $\mathrm{CD}^{+} / \mathrm{CD} 25^{+}$cells or Treg cells $\left(\mathrm{CD} 4^{+} / \mathrm{CD} 25^{+}\right.$and FoxP $3^{+}$) was performed using CellQuest Pro software (BD). The results are presented as the mean percentage of $\mathrm{CD}^{+}$cells \pm standard error of the mean.

2.8. Phagocytosis. Blood cell phagocytosis was assessed by PHAGOTEST $^{\mathrm{TM}}$ (BD Biosciences, Warsaw, Poland) according to the manufacturer's protocol. $100 \mu \mathrm{l}$ of whole blood (collected in heparin tube) was used per test. Blood was incubated for $10 \mathrm{~min}$. With opsonized GFP-stained E. coli in control $\left(0^{\circ} \mathrm{C}\right)$ and experimental $\left(37^{\circ} \mathrm{C}\right)$ conditions, then quenching solution was added, and red blood cells were lysed in lysing solution. Level of phagocytosis (in granulocytes or monocytes) was measured by flow cytometry (FACSCalibur). The results are presented as the mean \% of cells (granulocytes or monocytes) containing phagocytized E. coli \pm standard error of the mean.

2.9. Oxidative Burst. Quantitative determination of leukocyte oxidative burst was performed in heparinized whole blood using PHAGOBURST ${ }^{\mathrm{TM}}$ assay (BD Biosciences, Warsaw, Poland) according to the manufacturer's protocol, with our own modification. Standard kit contains unlabeled opsonized E. coli bacteria as particulate stimulus, the protein kinase $\mathrm{C}$ ligand phorbol 12-myristate 13-acetate (PMA) as high stimulus, and the chemotactic peptide $\mathrm{N}$-formyl-Met-Leu-Phe (fMLP) as low physiological stimulus. We modified protocol by replacing PMA with the zymosan $(20 \mu \mathrm{g} / \mathrm{ml})$. Oxidative burst analysis was performed by flow cytometry (FACSCalibur). The results are presented as the mean \% of cells (granulocytes or monocytes) with oxidative burst \pm standard error of the mean.

2.10. Statistical Analysis. Statistical evaluation of the results obtained, from the control and experimental groups, was performed using unpaired $t$-tests and one- or two-way analysis of the variance, followed by the Tukey test or Bonferroni correction (in the case of a normal distribution) or nonparametric Kruskal-Wallis and Mann-Whitney $U$ tests (in the case of abnormal distribution). Assessment of the distribution of the data was evaluated using the Shapiro-Wilk test. GraphPad Prism software was used to carry out these tests (version 5; GraphPad Software Inc., La Jolla, CA, USA). $P<0.05$ was considered as statistically significant.

\section{Results}

3.1. Hematological Analysis. There was no significant difference in RBC, HCT, MCH, MCHC, RDW\%, RDWa, PLT, and MPV parameters between control and experimental groups. The offspring of mice fed during pregnancy and lactation with water extract of Rhodiola kirilowii exhibited slightly higher (about $4 \%, P=0.0325$, statistically significant) concentration of hemoglobin in blood in comparison to control group. There was also a slight but statistically significant $(P=0.0354)$ difference between RKW and RKW-A group in mean corpuscular volume. The results of hematological analysis are presented in Table 1.

3.2. WBC Analysis. Water and hydroalcoholic extract of Rhodiola kirilowii did not affect the total number of WBC populations (lymphocytes, monocytes, and granulocytes) or percentage of monocytes in blood when compared to control group. Both extracts of Rhodiola kirilowii decreased proportion of lymphocytes and increased proportion of granulocytes. However, statistically significant differences (approximately $7 \%$ for lymphocytes $(P=0.0288)$ and $12 \%$ for granulocytes $(P=0.0243))$ were observed for water extract of Rhodiola kirilowii. Described results are presented in Table 2.

3.3. Lymphocyte Phenotyping. The offspring of mice that received Rhodiola kirilowii extracts during pregnancy and nursing period exhibited significantly lower percentage of $\mathrm{CD}^{+}$(approximately RKW-14\%, $P=0.0434$; RKW-A-10\%, 
TABLE 1: Blood hematological analysis. Analysis was performed in the offspring of mothers fed during pregnancy and lactation with water (RKW) or hydroalcoholic (RKW-A) extract of Rhodiola kirilowii. Bold font indicates statistically significant differences in comparison to control group $(P<0.05)$.

\begin{tabular}{lccccccc}
\hline \multirow{2}{*}{ Parameters } & \multirow{2}{*}{ Unit } & \multicolumn{2}{c}{ Control } & \multicolumn{2}{c}{ RKW } & \multicolumn{2}{c}{ RKW-A } \\
& & Mean & SEM & Mean & SEM & Mean & SEM \\
\hline RBC & $\times 10^{12} / 1$ & 10.3 & 0.1 & 10.5 & 0.1 & 10.3 & 0.1 \\
HGB & $\mathrm{g} / \mathrm{dl}$ & 15.4 & 0.2 & $\mathbf{1 6 . 0}$ & 0.2 & 15.7 & 0.2 \\
$\mathrm{HCT}$ & $\%$ & 55.8 & 0.8 & 57.1 & 0.8 & 56.0 & 0.7 \\
$\mathrm{MCV}$ & $\mathrm{fl}$ & 54.2 & 0.3 & 54.1 & 0.3 & 54.4 & 0.3 \\
$\mathrm{MCH}$ & $\mathrm{pg}$ & 15.3 & 0.1 & 15.4 & 0.1 & 15.3 & 0.1 \\
$\mathrm{MCHC}$ & $\mathrm{g} / \mathrm{dl}$ & 28.3 & 0.2 & 28.5 & 0.1 & 28.1 & 0.1 \\
$\mathrm{RDW}$ & $\%$ & 20.6 & 0.4 & 20.5 & 0.6 & 20.4 & 0.3 \\
$\mathrm{RDWa}$ & $\mathrm{fl}$ & 39.1 & 0.5 & 38.4 & 0.6 & 39.1 & 0.3 \\
$\mathrm{PLT}$ & $\times 10^{9} / 1$ & 510.1 & 47.8 & 521.6 & 62.2 & 487.2 & 32.9 \\
$\mathrm{MPV}$ & $\mathrm{fl}$ & 5.8 & 0.2 & 5.9 & 0.1 & 6.1 & 0.2 \\
\hline
\end{tabular}

TABLE 2: Blood WBC analysis. Analysis was performed in the offspring of mothers fed during pregnancy and lactation with water (RKW) or hydroalcoholic (RKW-A) extract of Rhodiola kirilowii. Bold font indicates statistically significant differences in comparison to control group $(P<0.05)$.

\begin{tabular}{lccccccc}
\hline \multirow{2}{*}{ Parameters } & \multirow{2}{*}{ Unit } & \multicolumn{2}{c}{ Control } & \multicolumn{2}{c}{ RKW } & \multicolumn{2}{c}{ RKW-A } \\
& & Mean & SEM & Mean & SEM & Mean & SEM \\
\hline WBC & $\times 10^{9} / 1$ & 5.49 & 0.57 & 4.66 & 0.30 & 5.36 & 0.44 \\
& $\times 10^{9} / 1$ & 3.70 & 0.37 & 3.00 & 0.21 & 3.51 & 0.33 \\
Lymphocytes & $\%$ & 68.62 & 1.63 & $\mathbf{6 4 . 0 2}$ & 1.06 & 64.17 & 1.64 \\
& $\times 10^{9} / 1$ & 0.37 & 0.05 & 0.27 & 0.03 & 0.32 & 0.03 \\
Monocytes & $\%$ & 6.60 & 0.62 & 5.71 & 0.37 & 5.97 & 0.43 \\
& $\times 10^{9} / 1$ & 1.00 & 0.14 & 0.98 & 0.07 & 0.99 & 0.07 \\
Granulocytes & $\%$ & 17.82 & 1.10 & $\mathbf{2 1 . 4 9}$ & 1.11 & 19.91 & 1.66 \\
\hline
\end{tabular}

$P=0.0337)$ and $\mathrm{CD}^{+}$cells $(15 \%, P=0.0184$ and $13 \%$, $P=0.0116$, resp.) in comparison to control group. Feeding with RKW extract caused statistically significant decrease in percentage of CD8-positive cells $(P=0.0342)$ and increase in percentage of CD335-positive NK cells $(P=0.0134)$ in comparison to control. There was no significant change in B-cell percentage between all studied groups (Figure 1).

There were some slight changes in the total number of lymphocytes in each studied subpopulation; however, the differences between control and extract-fed groups were not statistically significant (Figure 2).

3.4. Treg Analysis. There was no statistically significant difference in the percentage of $\mathrm{CD} 4^{+} / \mathrm{CD} 25^{+}$and Treg cells between examined groups (Figure 3 ).

3.5. Phagocytosis. Both extracts of Rhodiola kirilowii significantly stimulated granulocyte phagocytosis as measured by the number of cells containing opsonized E. coli, when compared to control group. The stimulation was approximately
$7 \%$ higher in RKW group $(P=0.0311)$ and 6\% in RKW-A group $(P=0.0370)$. Interestingly, monocytes isolated from blood of the offspring, which mothers were fed during pregnancy and nursing with water extract of Rhodiola kirilowii, exhibited significantly lower level of monocyte phagocytosis (about 12\%, $P=0.0075$ ). Level of monocyte phagocytosis in RKW-A group remained unaffected (Figure 4).

3.6. Oxidative Burst. Similar to phagocytosis, extracts of Rhodiola kirilowii administrated to pregnant and lactating mice increased level of respiratory burst stimulated by exposure to E. coli or fMLP. The granulocytes from RKW group exposed to $E$. coli (strong stimulant of oxidative burst) showed approximately $26 \%$ enhancement $(P=0.0021)$ of oxidative burst when compared to control. Addition of low physiological stimulant, fMLP caused significant increase of granulocyte respiratory burst in both RKW and RKW-A groups ( $46 \%$ with $P=0.0010$ and $40 \%$ with $P=0.0066$, resp.). There was no significant change after zymosan (all groups) and E. coli (RKW-A group) exposure (Figure 5).

There was also no significant difference between control and Rhodiola kirilowii groups in monocyte oxidative burst after E. coli and fMLP stimulation. The statistically significant decrease of oxidative burst occurred only after zymosan stimulation and only in RKW (around 28\%, $P=0.0098$ ) group. There was no difference in RKW-A group (Figure 5).

\section{Discussion}

The immune system of fetus develops slowly during pregnancy and rapidly during the first period after birth [26]. Proper functioning of the immune system is essential for defense against pathogens and regulation of homeostasis. Our previous experiments in mouse model showed that Rhodiola kirilowii extract affects certain parameters of pregnancy such as litter size and number of females without progeny [23, 27].

In this study, we showed that the Rhodiola kirilowii extracts administrated to the mouse mothers during lactation and nursing did not affect the hematological parameters. The only exception was a slight but statistically significant increase in the concentration of hemoglobin in the group supplemented with an aqueous extract of Rhodiola kirilowii. Similar results were obtained by Gupta et al. [28] with another Rhodiola species. In these studies, rats treated with Rhodiola imbricate did not show significant changes in the blood. Also, Senthilkumar et al. [29] had not observed any significant differences in blood parameters of Wistar rats after administration of the acetone extract of Rhodiola imbricata (200 and $400 \mathrm{mg} / \mathrm{kg}$ ). Moreover, Rhodiola imbricata supplementation showed hepatoprotective properties against paracetamol-induced liver toxicity.

There are many factors, which are able to modify (increase or decrease) the population of WBC cells. Such biologically active compounds are probably present in water extract and hydroalcoholic extract of Rhodiola kirilowii. In our previous study, we showed that both extracts of Rhodiola kirilowii contain kaempferol, epicatechin quercetin, $(+)$-catechin, salidroside, fisetin, naringenin, luteolin, p- 

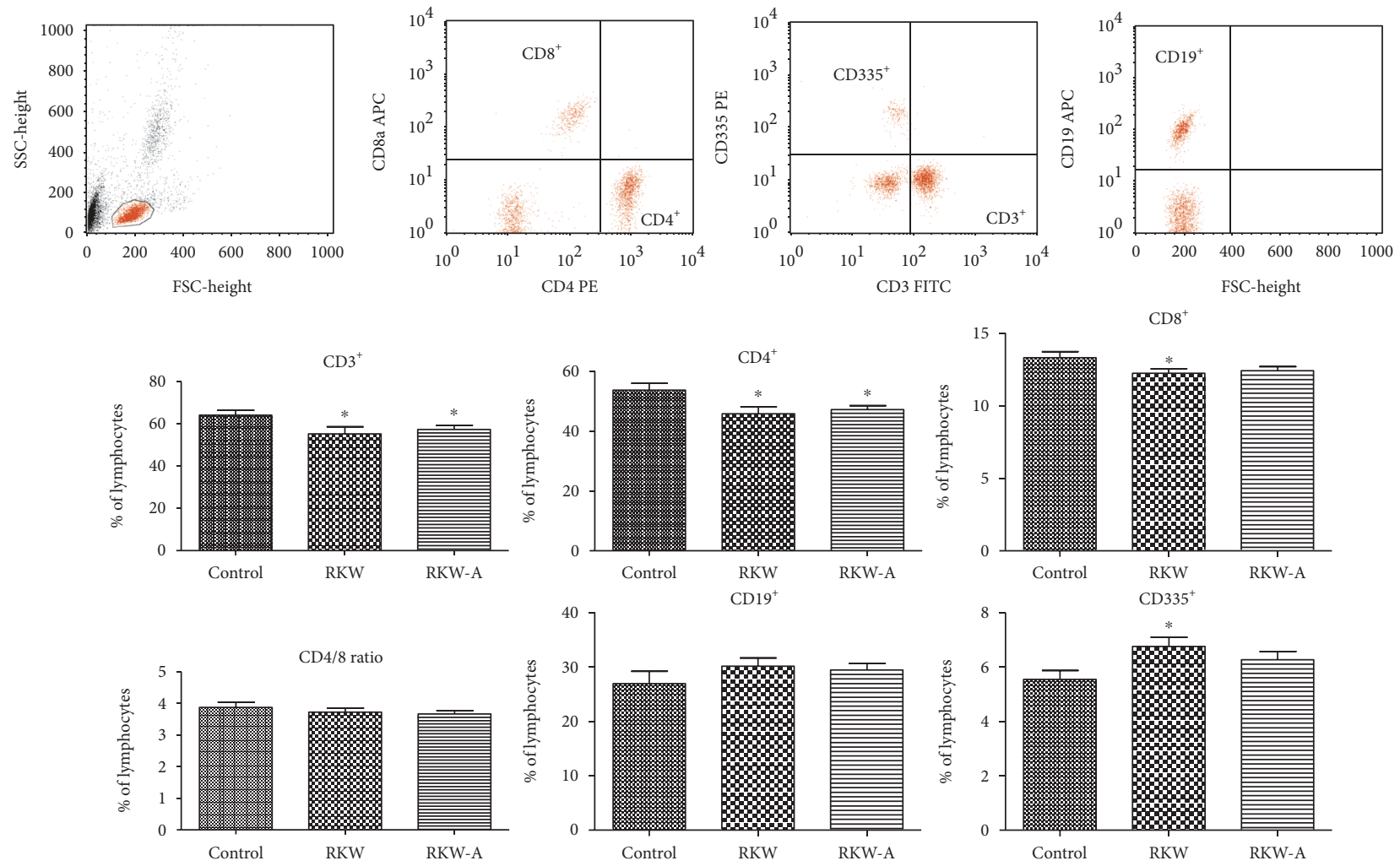

Figure 1: Blood lymphocyte phenotype analysis (mean \% \pm SEM). Analysis was performed in the offspring of mothers fed during pregnancy and lactation with water (RKW) or hydroalcoholic (RKW-A) extract of Rhodiola kirilowii. ${ }^{*} P<0.05$.
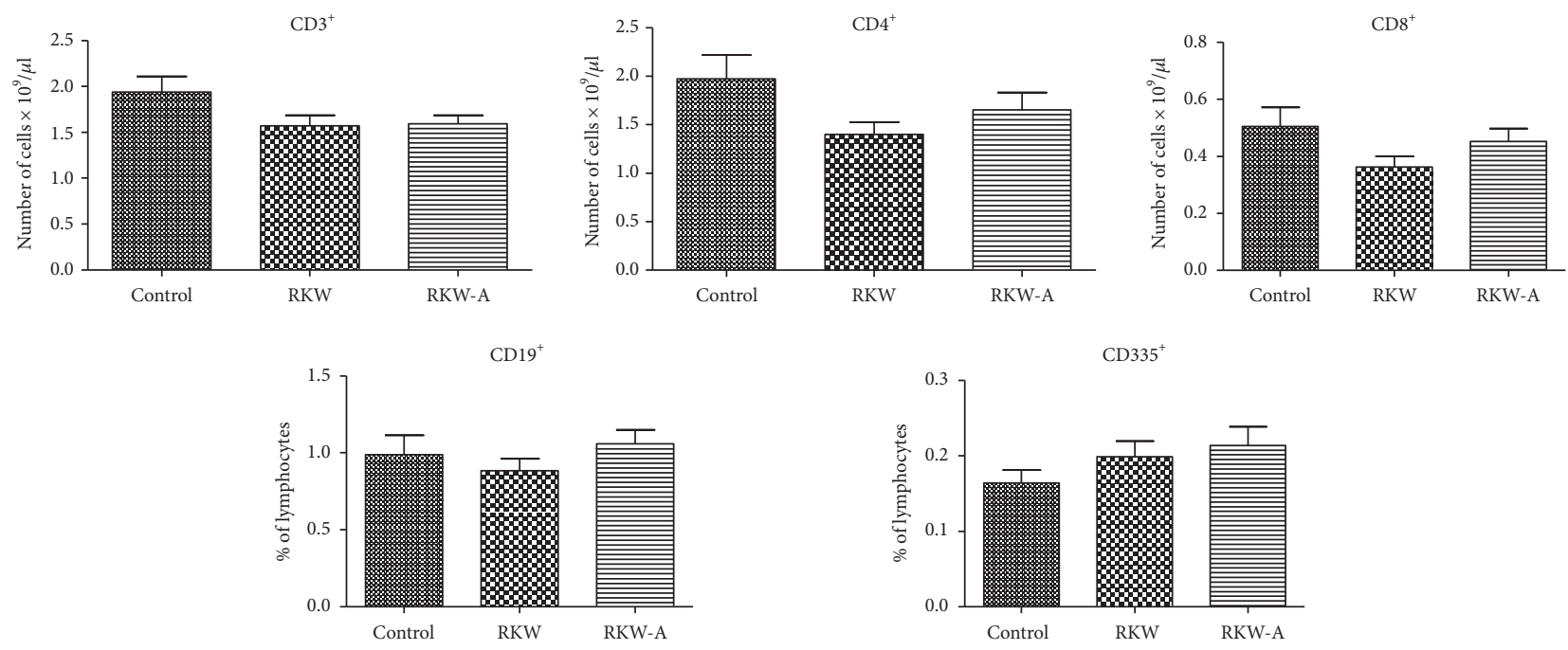

Figure 2: Blood lymphocyte phenotype analysis (cell number per $\mu \mathrm{l}$ ). Analysis was performed in the offspring of mothers fed during pregnancy and lactation with water (RKW) or hydroalcoholic (RKW-A) extract of Rhodiola kirilowii.

coumaric acid, ellagic, acid epigallocatechin, ferulic and chlorogenic acid, epicatechin gallate, and epigallocatechin gallate [27]. Some of these compounds were also found in sera of mouse mothers [30]. It is known that some of biological compounds found in Rhodiola kirilowii may affect the number, percentage distribution, and activity of WBC populations. For example, the salidroside modulates mouse inflammatory responses, both in the number of immunological cells and in secretion of inflammatory cytokines [31]. It also positively affects bone marrow (BM) function by modulating the number of peripheral white blood cells in bone marrow-depressed mice [32].

We showed here that the offspring of mice that received Rhodiola kirilowii extracts during pregnancy and nursing 

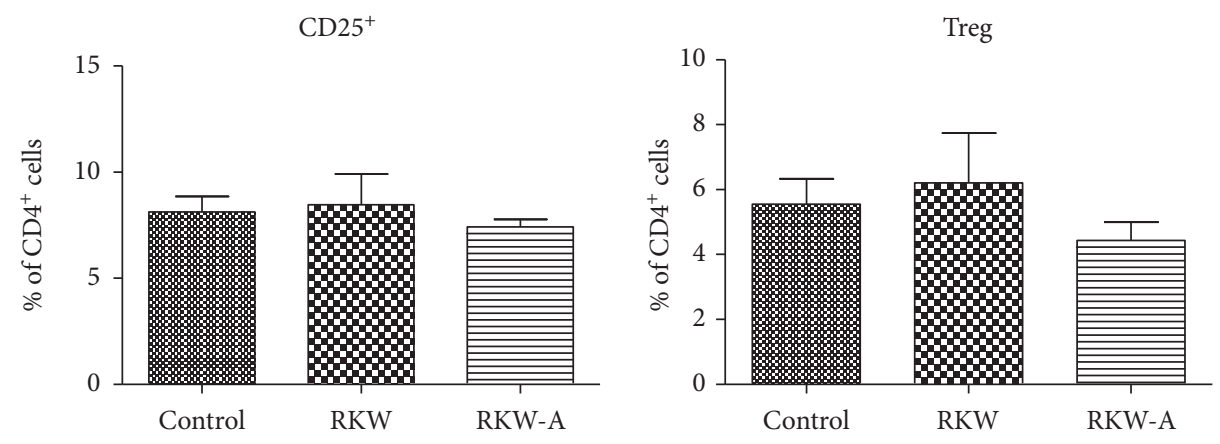

FIGURE 3: Treg cells in peripheral blood. Analysis was performed in the offspring of mothers fed during pregnancy and lactation with water (RKW) or hydroalcoholic (RKW-A) extract of Rhodiola kirilowii.
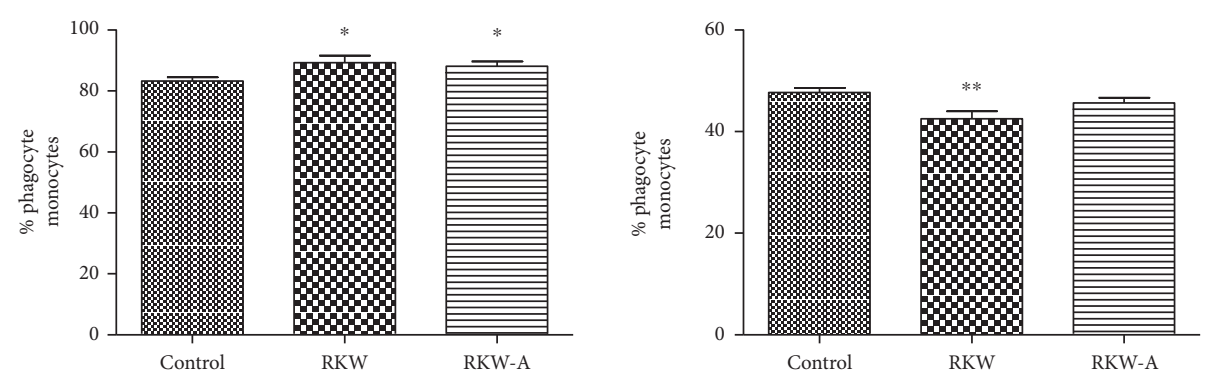

FIGURE 4: Blood cell phagocytosis. Analysis was performed in the offspring of mothers fed during pregnancy and lactation with water (RKW) or hydroalcoholic (RKW-A) extract of Rhodiola kirilowii. ${ }^{*} P<0.05 ;{ }^{* *} P<0.01$.
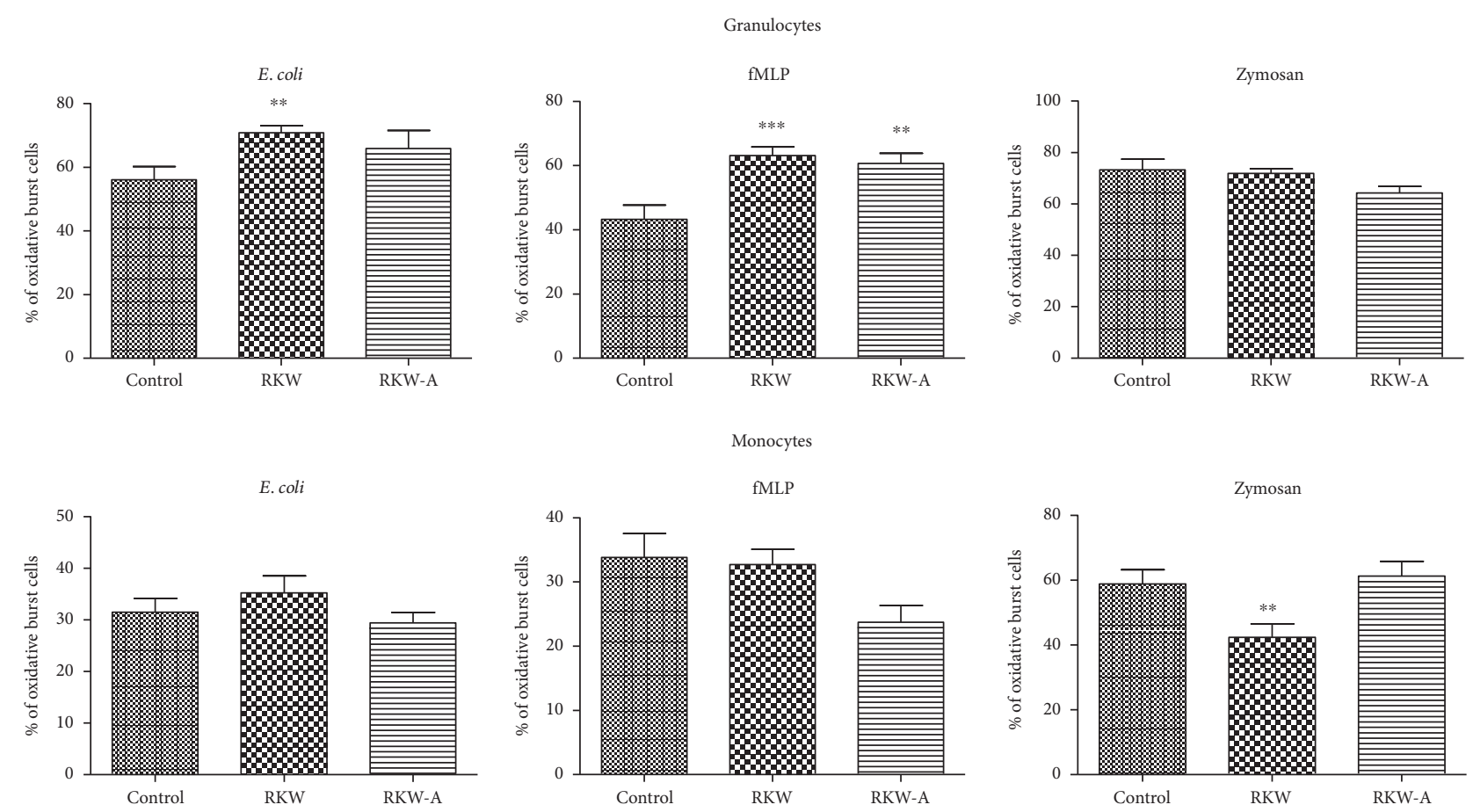

FIGURE 5: Oxidative burst. Analysis was performed in the offspring of mothers fed during pregnancy and lactation with water (RKW) or hydroalcoholic (RKW-A) extract of Rhodiola kirilowii. ${ }^{* *} P<0.01{ }^{* * *} P<0.001$.

period exhibited statistically significant lower percentage of $\mathrm{CD}^{+}{ }^{+} \mathrm{T}$-cells and decrease of $\mathrm{CD}^{+}{ }^{+} \mathrm{T}$-cells, in comparison to control group. $\mathrm{CD}^{+} \mathrm{T}$-cells are important for adaptive immunity response [33]. These cells recognize $\mathrm{MHC}$ class II protein complex and are involved in the induction and the restraint of most immunological functions. A deficiency 
of $\mathrm{CD} 4^{+} \mathrm{T}$-cells causes significant attenuation of adaptive response. This is especially visible in HIV patients where reduction of $\mathrm{CD}^{+}$T-cells causes immunological failure [34]. To confirm this conjectures, in our previous studies [24], we checked the functionality of the components of adaptive response in the progeny after Rhodiola extract administration. Spleen cell number and spleen cell phenotype $\left(\mathrm{CD}^{+}, \mathrm{CD}^{+}, \mathrm{CD} 8^{+}\right.$, and $\left.\mathrm{CD} 19^{+}\right)$were not affected after RKW treatment. Also, spleen cell proliferation (after lipopolysaccharide and phytohaemagglutinin) was not affected; however, we noted significant reduction of spleen cell proliferation after concanavalin A stimulation in RKW group, which is somewhat worrying. Reduction in the percentage of $\mathrm{CD}^{+}$cells observed in previous work was also not associated with the significant reduction in the total number of $\mathrm{CD}^{+}$cells. $\mathrm{CD}^{+}$T-cells cooperate with B-cells and together promote strength and duration of adaptive immune response. Here, we showed that there was no significant difference in B-cell percentage and number between Rhodiola-treated and control groups. All these data indicated that RKW extract did not have negative effect on the adaptive functionality. Administration of SRBC to the progeny which mothers were fed with RKW extracts during pregnancy and nursing did not influence antibody production [24]. In contrast, the hydroalcoholic extract of Rhodiola kirilowii significantly decreased the level of antibodies in serum (SRBC test) and significantly reduced spleen cell proliferation. This suggests that about $33 \%$ increase of polyphenols in mothers' diet (differences between RKW and RKW-A) significantly affects adaptive immune response.

Similar situation (significantly lower percentage of $\mathrm{CD} 8^{+}$ T-cells in the blood) was observed in RKW group. Decreased level of both T-cell populations suggests their faulty production in bone marrow or faulty development in the thymus. However, the results of our previous studies indicated that feeding pregnant and lactating mothers with water or hydroalcoholic extract of Rhodiola kirilowii decreased thymus $\mathrm{T}$ lymphocyte apoptosis and did not significantly affect IL-7 expression in progeny's thymocytes [35]. Similar results were reported by Liu et al. [36] in the model of septic rats after administration of Rhodiola rosea $(50 \mathrm{mg} / \mathrm{kg}$ body weight). These changes correlated with downregulation of tumor necrosis factor- $\alpha$-induced protein 8 -like 2 . Taken together, these results indicate that the extracts from Rhodiola help to preserve thymus function in the progeny of treated animals. We believe that a decreased level of $\mathrm{CD}^{+}{ }^{+} \mathrm{T}$-cells in the blood may be a consequence of the increased mobility of these cells. Our previous studies showed significantly higher number of $\mathrm{CD} 8^{+} \mathrm{T}$-cells in the spleen of the progeny whose mothers were fed with Rhodiola kirilowii extracts [37]. Moreover, $\mathrm{CD} 8^{+} \mathrm{T}$-positive cells were found in the nontypical location within the spleen, that is, not only in the PALS but also in the B-cell follicle and in the red pulp.

The lack of effect of RKW extracts on the adaptive immunity is also confirmed by the results of Treg cell analysis. Our present study showed that there was no significant difference in the total number and percentage of regulatory T-cells (Treg) between control and Rhodiola-treated groups. Also, $\mathrm{Xu}$ et al. [38] did not observe significant changes in the ratio of circulating Tregs or Treg cell differentiation in the mice treated with Rhodiola rosea. These results should be seen as positive outcome of the treatment, because changes in the number and percentage of Tregs may be one of the reasons for the development of autoimmune, allergic, or malignant diseases [39].

Another positive outcome of the Rhodiola treatment is visible in the behavior of NK cells. We observed significant increase of NK cell percentage in the blood of mice whose mothers were fed during pregnancy and lactation with water extract of Rhodiola kirilowii. The NK cells exhibit cytotoxic properties and are the part of the innate immune system. The NK cells survey the body for aberrant expression of MHC class I molecules and stress markers on the autologous cells [40]. Increased number and activity of NK cells are especially desirable for negative regulation of growth and metastasis of tumors. Lee et al. [41] showed that NK cells isolated from human blood reduce systemic metastasis and inhibit development of glioblastoma cells in nude mouse. Also, Diwaker et al. [42] noted increased number of NK cells after Rhodiola treatment in dengue-virus-infected human PBMCs.

Phagocytosis is a major process in which granulocytes and monocytes eliminate pathogens [43]. Several studies showed that the granulocytes exhibit higher level of phagocytosis than monocytes $[44,45]$. Filias et al. [46] showed that phagocytotic ability of granulocytes and monocytes in the neonates is fully functional few days after birth. Although the standard isolation procedures do not affect phagocytosis of Escherichia coli bacteria [47], the isolation procedures may affect the granulocyte respiratory burst but only after anti-CD15 antibody-conjugated microbead isolation (positive selection).

We observed an increased level of phagocytic activity in granulocytes from Rhodiola extract group. Additionally, there were changes in oxidative burst after strong (E. coli) and weak (fMLP) stimulation. RKW groups showed higher stimulation of phagocytosis and oxidative burst than RKWA groups. There is strong evidence that herbs from Rhodiola species stimulate innate immunity by enhancement of phagocytosis. These effects were found in several mammalian species (mice, rats, and pigs) after administration of Rhodiola quadrifida or Rhodiola rosea [20,21]. Administration of Rhodiola kirilowii to the mouse mothers during pregnancy and nursing significantly improves activity of innate immunity cells of their offspring. These are very promising results; however, further studies are needed to elucidate the mechanisms of Rhodiola action in pregnancy and lactation.

Cytokines are hormone-like messengers regulating development and functionality of both adaptive and innate immune responses [48, 49]. There are several studies describing the effects of Rhodiola spp. on the cytokine secretion. Lin et al. [50] showed increased secretion of both Th1- and Th2pattern cytokines in a dose- and time-dependent manner after Rhodiola rosea treatment. The Rhodiola rosea supplementation enhanced production of interferon (IFN) $\beta$ and other cytokines, including IL- $1 \beta$, TNF- $\alpha$, IL- 6 , and IL-8, in the monocytes infected with dengue virus [42]. We also checked the expression of cytokines in the serum of the progeny which mothers were fed during pregnancy and 
lactation with the RK extracts. The RKW and RKW-A extracts supplementation did not change the expression of IFN $\gamma$, IL-2, IL- 4 or IL- 6 in progeny sera compared with the control. However, TNF- $\alpha$ and IL-10 expression was higher in the progeny of mice fed with RKW-A extract [24]. The RKW administration lowered the concentration of interleukin-17a. It is known that IL-17a induces and mediates the proinflammatory responses and promotes production of IL- 6 , TNF- $\alpha$, GCF, GM-CSF, IL- $1 \beta$, TGF- $\beta$, and IL-8 $[51,52]$.

Our present and previously published studies tried to assess, which one of the two extracts, water (RKW) or hydroalcoholic (RKW-A) of Rhodiola kirilowii when given during pregnancy and lactation period that are less detrimental to the health of the offspring. We showed that the water extract (RKW) is less detrimental to the health of the offspring. This conclusion is based on the following data:

(i) The offspring of mothers fed during pregnancy and nursing period with water extract (RKW) of Rhodiola kirilowii exhibits stimulation of innate immune response (granulocytes and NK cells) and slight decrease of monocyte activity (present work), without changes in adaptive immune response (SRBC blood test). In contrast, feeding with RKW-A impaired adaptive response [24].

(ii) There were no significant differences in spleen cell response after lipopolysaccharide and phytohaemagglutinin, and there was a decreased response after concanavalin A stimulation in RKW group. In contrast, feeding with RKW-A diminished response to all these stimulants [24].

(iii) Both extracts protected thymus cells from apoptosis [35].

(iv) The offspring of RKW-fed group, in contrast to RKW-A-fed group, had no significant differences in kidney structure and function [53]. In addition, mice from RKW-A group had higher level of angiogenic factors (VEGF and bFGF) in serum [30].

(v) There were no neonatal deaths in RKW and control groups and several neonatal deaths in RKW-A group [23].

Taking together, these results suggest that water extract of Rhodiola kirilowii is safer to use during pregnancy and lactation.

\section{Conclusions}

Long-term supplementation of mouse mothers during pregnancy and lactation with water extract or hydroalcoholic extract of Rhodiola kirilowii affects some parameters of the immune system of their progeny. These treatments increase the number and activity of innate immune cells in blood and slightly decrease the percentage of T-cells (mainly $\mathrm{CD}^{+}$). The results obtained in the present work provide evidence that plant-derived biologically active compounds administrated to the mothers during pregnancy and nursing period may significantly and permanently affect the immune response of their offspring. These results can be useful for creation of guidelines for pregnant and lactating women's diet.

\section{Conflicts of Interest}

The authors certify that there is no conflict of interests with any financial organization regarding the material discussed in the paper.

\section{Acknowledgments}

The study was supported by the National Centre of Science, Grant no. 2012/05/B/NZ 7/03219.

\section{References}

[1] W. Jędrychowski, A. Gałaś, R. Whyatt, and F. Perera, "The prenatal use of antibiotics and the development of allergic disease in one year old infants. A preliminary study," International Journal of Occupational Medicine and Environmental Health, vol. 19, no. 1, pp. 70-76, 2006.

[2] A. Hecht, "Drug safety labeling for doctors," FDA Consumer, vol. 13, no. 8, pp. 12-13, 1979.

[3] E. Skopińska-Różewska, M. Mościcka-Wesołowska, A. Wasiutyński, J. Małdyk, M. Malejczyk, and J. Pazdur, "Lymphatic system of mice born from mothers treated with ampicillin or cloxacillin during gestation," Archivum Immunologiae et Therapiae Experimentalis, vol. 34, no. 2, pp. 203-209, 1985.

[4] B. Włodarska, J. Bany, M. Marczak et al., "Altered immune reactivity of mice born from mothers treated with ampicillin during gestation," Folia Biologica, vol. 33, no. 3, pp. 211-215, 1987.

[5] N. T. Mueller, E. Bakacs, J. Combellick, Z. Grigoryan, and M. G. Dominguez-Bello, "The infant microbiome development: mom matters," Trends in Molecular Medicine, vol. 21, no. 2, pp. 109-117, 2015.

[6] T. F. Landers, B. Cohen, T. E. Wittum, and E. L. Larson, "A review of antibiotic use in food animals: perspective, policy, and potential," Public Health Reports, vol. 127, no. 1, pp. 4-22, 2012.

[7] C. L. Ventola, "The antibiotic resistance crisis: part 1: causes and threats," Pharmacy and Therapeutics, vol. 40, no. 4, pp. 277-283, 2015.

[8] A. G. Mathew, R. Cissell, and S. Liamthong, "Antibiotic resistance in bacteria associated with food animals: a United States perspective of livestock production," Foodborne Pathogens and Disease, vol. 4, no. 2, pp. 115-133, 2007.

[9] M. Grech-Baran, K. Sykłowska-Baranek, and A. Pietrosiuk, "Approaches of Rhodiola kirilowii and Rhodiola rosea field cultivation in Poland and their potential health benefits," Annals of Agricultural and Environmental Medicine, vol. 22, no. 2, pp. 281-285, 2015.

[10] M. L. Chuang, T. C. Wu, Y. T. Wang et al., "Adjunctive treatment with Rhodiola crenulata in patients with chronic obstructive pulmonary disease - a randomized placebo controlled double blind clinical trial," PLoS One, vol. 10, no. 6, article e0128142, 2015. 
[11] L. Yu, Y. Qin, Q. Wang et al., "The efficacy and safety of Chinese herbal medicine, Rhodiola formulation in treating ischemic heart disease: a systematic review and meta-analysis of randomized controlled trials," Complementary Therapies in Medicine, vol. 22, no. 4, pp. 814-825, 2014.

[12] K. P. Mishra, L. Ganju, and S. B. Singh, "Anti-cellular and immunomodulatory potential of aqueous extract of Rhodiola imbricata rhizome," Immunopharmacology and Immunotoxicology, vol. 34, no. 3, pp. 513-518, 2012.

[13] J. L. Cui, T. T. Guo, Z. X. Ren, N. S. Zhang, and M. L. Wang, "Diversity and antioxidant activity of culturable endophytic fungi from alpine plants of Rhodiola crenulata, R. angusta, and R. sachalinensis," PLoS One, vol. 10, no. 3, article e0118204, 2015.

[14] G. Zuo, Z. Li, L. Chen, and X. Xu, "Activity of compounds from Chinese herbal medicine Rhodiola kirilowii (Regel) Maxim against HCV NS3 serine protease," Antiviral Research, vol. 76, no. 1, pp. 86-92, 2007.

[15] Y. C. Wong, M. Zhao, Y. Y. Zong, C. Y. Chan, and C. T. Che, "Chemical constituents and anti-tuberculous activity of root of Rhodiola kirilowii," Zhongguo Zhong Yao Za Zhi, vol. 33, no. 13, pp. 1561-1565, 2008.

[16] K. Khanna, K. P. Mishra, L. Ganju, and S. B. Singh, "Golden root: a wholesome treat of immunity," Biomedicine \& Pharmacotherapy, vol. 87, pp. 496-502, 2017.

[17] E. Skopinska-Różewska, M. Bychawska, B. Białas-Chromiec, and E. Sommer, "The in vivo effect of Rhodiola rosea and Rhodiola quadrifida hydro-alcoholic extracts on chemokinetic activity of spleen lymphocytes in mice," Central European Journal of Immunology, vol. 34, no. 1, pp. 42-45, 2009.

[18] R. Wójcik, A. K. Siwicki, E. Skopińska-Rózewska, A. Wasiutyński, E. Sommer, and M. Furmanowa, "The effect of Chinese medicinal herb Rhodiola kirilowii extracts on cellular immunity in mice and rats," Polish Journal of Veterinary Sciences, vol. 12, no. 3, pp. 399-405, 2009.

[19] R. Zdanowski, S. Lewicki, E. Skopińska-Różewska, W. Buchwald, P. M. Mrozikiewicz, and W. Stankiewicz, "Alcohol- and water-based extracts obtained from Rhodiola rosea affect differently the number and metabolic activity of circulating granulocytes in Balb/c mice," Annals of Agricultural and Environmental Medicine, vol. 21, no. 1, pp. 120-123, 2014.

[20] E. Skopińska-Rózewska, R. Wójcik, A. K. Siwicki et al., “The effect of Rhodiola quadrifida extracts on cellular immunity in mice and rats," Polish Journal of Veterinary Sciences, vol. 11, no. 2, pp. 105-111, 2008.

[21] A. K. Siwicki, E. Skopińska-Rożewska, M. Hartwich et al., “The influence of Rhodiola rosea extracts on nonspecific and specific cellular immunity in pigs, rats and mice," Central European Journal of Immunology, vol. 32, no. 2, pp. 84-91, 2007.

[22] E. Skopińska-Różewska, W. Stankiewicz, R. Zdanowski et al., "The in vivo effect of Rhodiola quadrifida extracts on the antibody production, on the blood leukocytes subpopulations and on the bacterial infection in mice," Central European Journal of Immunology, vol. 37, no. 2, pp. 140-144, 2012.

[23] R. Zdanowski, S. Lewicki, K. Sikorska et al., "The influence of aqueous and hydro-alcoholic extracts of roots and rhizomes of Rhodiola kirilowii on the course of pregnancy in mice," Central European Journal of Immunology, vol. 39, no. 4, pp. 471-475, 2014.

[24] S. Lewicki, B. J. Bałan, E. Skopińska-Różewska et al., "Modulatory effects of feeding pregnant and lactating mice Rhodiola kirilowii extracts on the immune system of offspring,"
Experimental and Therapeutic Medicine, vol. 12, no. 5, pp. 3450-3458, 2016.

[25] B. Kalicki, S. Lewicki, W. Stankiewicz et al., "Examination of correlation between vitamin D3 (25-OHD3) concentration and percentage of regulatory $\mathrm{T}$ lymphocytes (FoxP3) in children with allergy symptoms," Central European Journal of Immunology, vol. 38, no. 1, pp. 70-75, 2013.

[26] G. Mor and I. Cardenas, "The immune system in pregnancy: a unique complexity," American Journal of Reproductive Immunology, vol. 63, no. 6, pp. 425-433, 2010.

[27] S. Lewicki, W. Stankiewicz, E. Skopińska-Różewska et al., "Spleen content of selected polyphenols, splenocytes morphology and function in mice fed Rhodiola kirilowii extracts during pregnancy and lactation," Polish Journal of Veterinary Sciences, vol. 18, no. 4, pp. 847-855, 2015.

[28] V. Gupta, S. Saggu, R. K. Tulsawani, R. C. Sawhney, and R. Kumar, "A dose dependent adaptogenic and safety evaluation of Rhodiola imbricata Edgew, a high altitude rhizome," Food and Chemical Toxicology, vol. 46, no. 5, pp. 1645-1652, 2008.

[29] R. Senthilkumar, R. Chandran, and T. Parimelazhagan, "Hepatoprotective effect of Rhodiola imbricata rhizome against paracetamol-induced liver toxicity in rats," Saudi Journal of Biological Sciences, vol. 21, no. 5, pp. 409-416, 2014.

[30] R. Zdanowski, E. Skopińska-Różewska, J. Wilczak, A. Borecka, A. Lewicka, and S. Lewicki, "Different effects of feeding pregnant and lactating mice Rhodiola kirilowii aqueous and hydro-alcoholic extracts on their serum angiogenic activity and selected polyphenols content," Central European Journal of Immunology, vol. 42, pp. 17-23, 2017.

[31] S. Guan, Y. Xiong, B. Song et al., "Protective effects of salidroside from Rhodiola rosea on LPS-induced acute lung injury in mice," Immunopharmacology and Immunotoxicology, vol. 34, no. 4, pp. 667-672, 2012.

[32] X. Zhang, B. Zhu, S. Jin, S. Yan, and Z. Chen, "Effects of salidroside on bone marrow matrix metalloproteinases of bone marrow depressed anemic mice," Sheng Wu Yi Xue Gong Cheng Xue Za Zhi, vol. 23, no. 6, pp. 1314-1319, 2006.

[33] R. V. Luckheeram, R. Zhou, A. D. Verma, and B. Xia, "CD4 ${ }^{+} \mathrm{T}$ cells: differentiation and functions," Clinical and Developmental Immunology, vol. 2012, Article ID 925135, 12 pages, 2012.

[34] A. A. Okoye and L. J. Picker, "CD4 ${ }^{+}$T-cell depletion in HIV infection: mechanisms of immunological failure," Immunological Reviews, vol. 254, no. 1, pp. 54-64, 2013.

[35] K. Bień, S. Lewicki, R. Zdanowski, E. Skopinska-Różewska, and M. Krzyżowska, "Feeding pregnant and lactating mice Rhodiola kirilowii extracts helps to preserve thymus function of their adult progeny," Polish Journal of Veterinary Sciences, vol. 19, no. 3, pp. 581-587, 2016.

[36] M. W. Liu, M. X. Su, W. Zhang, L. M. Zhang, Y. H. Wang, and C. Y. Qian, "Rhodiola rosea suppresses thymus T-lymphocyte apoptosis by downregulating tumor necrosis factor- $\alpha$-induced protein 8-like-2 in septic rats," International Journal of Molecular Medicine, vol. 36, no. 2, pp. 386-398, 2015.

[37] S. Lewicki, P. Orłowski, M. Krzyżowska, A. Kiepura, E. Skopińska-Różewska, and R. Zdanowski, "The effect of feeding mice during gestation and nursing with Rhodiola kirilowii extracts or epigallocatechin on CD4 and CD8 cells number and distribution in the spleen of their progeny," Central European Journal of Immunology, vol. 42, no. 1, pp. 10-16, 2017. 
[38] X. Xu, P. Li, P. Zhang et al., "Differential effects of Rhodiola rosea on regulatory $\mathrm{T}$ cell differentiation and interferon- $\gamma$ production in vitro and in vivo," Molecular Medicine Reports, vol. 14, no. 1, pp. 529-536, 2016.

[39] D. Wolf, S. Sopper, A. Pircher, G. Gastl, and A. M. Wolf, "Treg(s) in Cancer: Friends or foe?," Journal of Cellular Physiology, vol. 230, no. 11, pp. 2598-2605, 2015.

[40] E. Vivier, E. Tomasello, M. Baratin, T. Walzer, and S. Ugolini, "Functions of natural killer cells," Nature Immunology, vol. 9, no. 5, pp. 503-510, 2008.

[41] S. J. Lee, W. Y. Kang, Y. Yoon et al., "Natural killer (NK) cells inhibit systemic metastasis of glioblastoma cells and have therapeutic effects against glioblastomas in the brain," $B M C$ Cancer, vol. 15, p. 1011, 2015.

[42] D. Diwaker, K. P. Mishra, L. Ganju, and S. B. Singh, "Rhodiola inhibits dengue virus multiplication by inducing innate immune response genes RIG-I, MDA5 and ISG in human monocytes," Archives of Virology, vol. 159, no. 8, pp. 19751986, 2014.

[43] D. C. Dale, L. Boxer, and W. C. Liles, "The phagocytes: neutrophils and monocytes," Blood, vol. 112, no. 4, pp. 935-945, 2008.

[44] R. T. Steigbigel, L. H. Lambert, and J. S. Remington, "Phagocytic and bactericidal properties of normal human monocytes," Journal of Clinical Investigation, vol. 53, no. 1, pp. 131-142, 1974.

[45] W. Hirt, T. Nebe, and C. Birr, "Phagotest and Bursttest (Phagoburst), test kits for study of phagocyte functions," Wiener Klinische Wochenschrift, vol. 106, no. 8, pp. 250-252, 1994.

[46] A. Filias, G. L. Theodorou, S. Mouzopoulou, A. A. Varvarigou, S. Mantagos, and M. Karakantza, "Phagocytic ability of neutrophils and monocytes in neonates," BMC Pediatrics, vol. 11, no. 29, 2011.

[47] L. Zhou, R. Somasundaram, R. F. Nederhof et al., "Impact of human granulocyte and monocyte isolation procedures on functional studies," Clinical and Vaccine Immunology, vol. 19, no. 7, pp. 1065-1074, 2012.

[48] J. L. Banyer, N. H. Hamilton, I. A. Ramshaw, and A. J. Ramsay, "Cytokines in innate and adaptive immunity," Reviews in Immunogenetics, vol. 2, no. 3, pp. 359-373, 2000.

[49] K. Newton and V. M. Dixit, "Signaling in innate immunity and inflammation," Cold Spring Harbor Perspectives in Biology, vol. 4, no. 3, article a006049, 2012.

[50] S. S. Lin, L. W. Chin, P. C. Chao et al., "In vivo Th1 and Th2 cytokine modulation effects of Rhodiola rosea standardised solution and its major constituent, salidroside," Phytotherapy Research, vol. 25, no. 11, pp. 1604-1611, 2011.

[51] W. Ouyang, J. K. Kolls, and Y. Zheng, "The biological functions of T helper 17 cell effector cytokines in inflammation," Immunity, vol. 28, no. 4, pp. 454-467, 2008.

[52] R. M. Onishi and S. L. Gaffen, "Interleukin-17 and its target genes: mechanisms of interleukin-17 function in disease," Immunology, vol. 129, no. 3, pp. 311-321, 2010.

[53] S. Lewicki, E. Skopińska-Różewska, B. J. Bałan et al., "Morpho-functional renal alterations in progeny of mice fed Rhodiola kirilowii extracts or epigallocatechin during pregnancy and lactation," Journal of Medicinal Food, vol. 20, no. 1, pp. 86-92, 2017. 


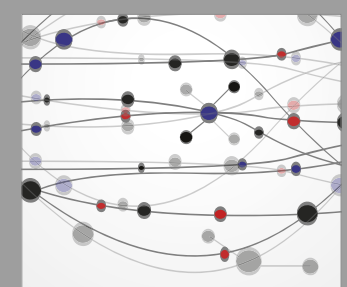

The Scientific World Journal
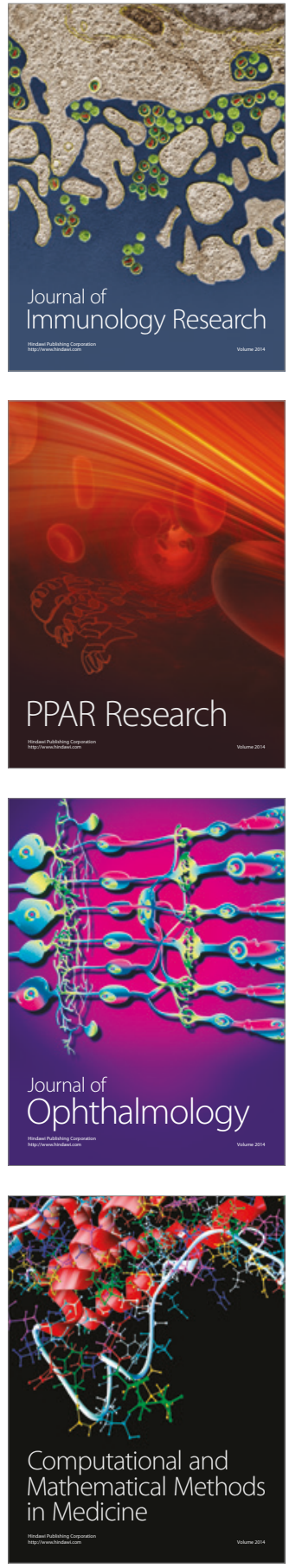

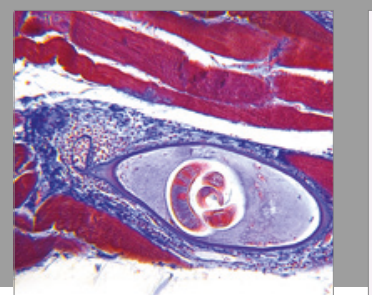

Gastroenterology Research and Practice
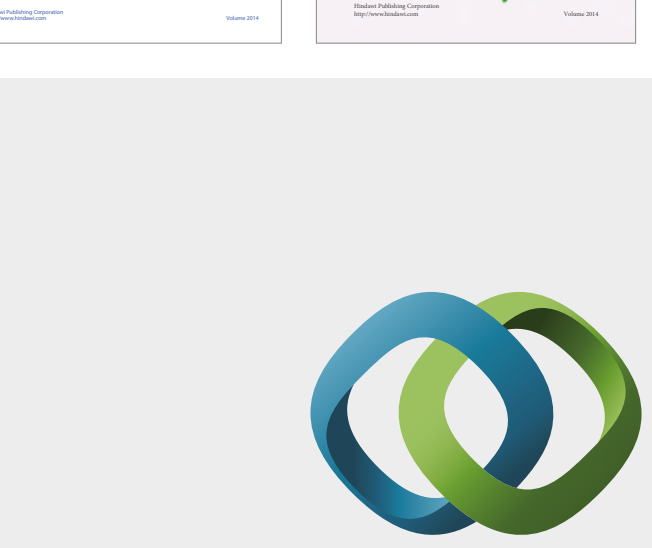

\section{Hindawi}

Submit your manuscripts at

https://www.hindawi.com
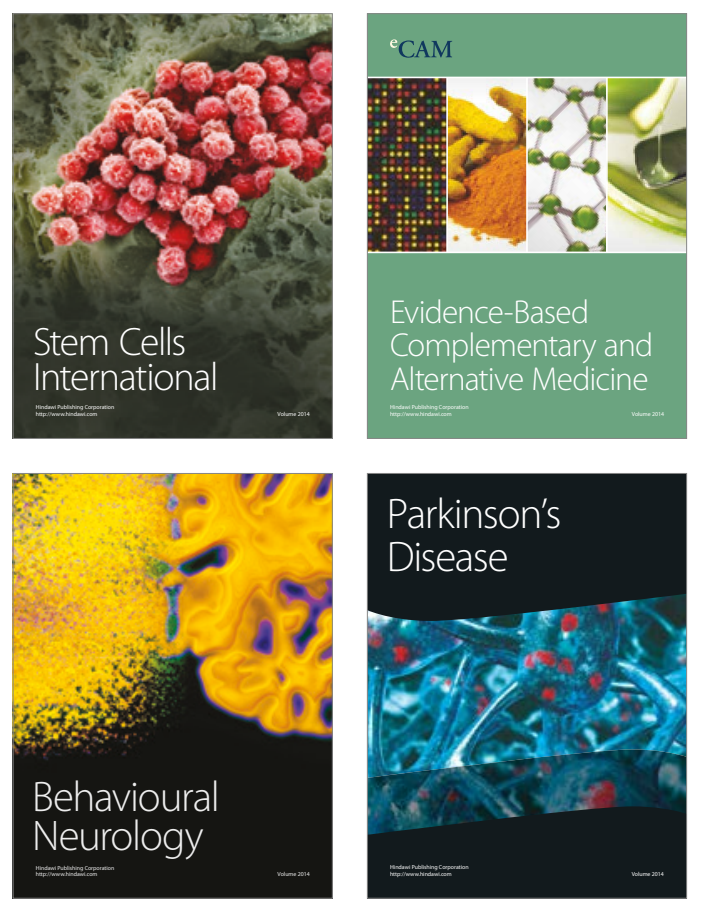
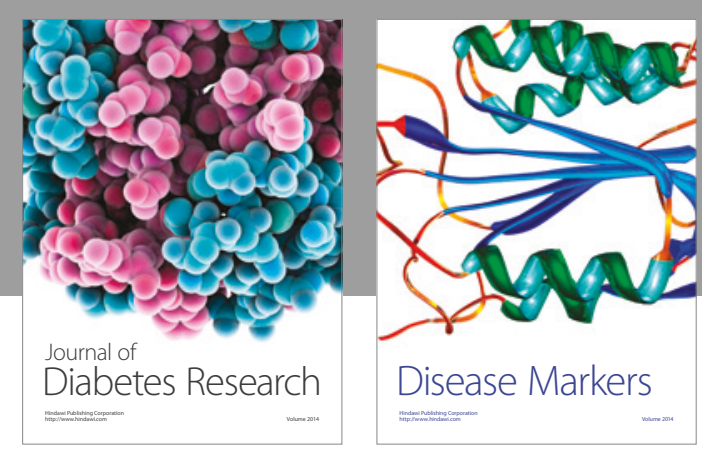

Disease Markers
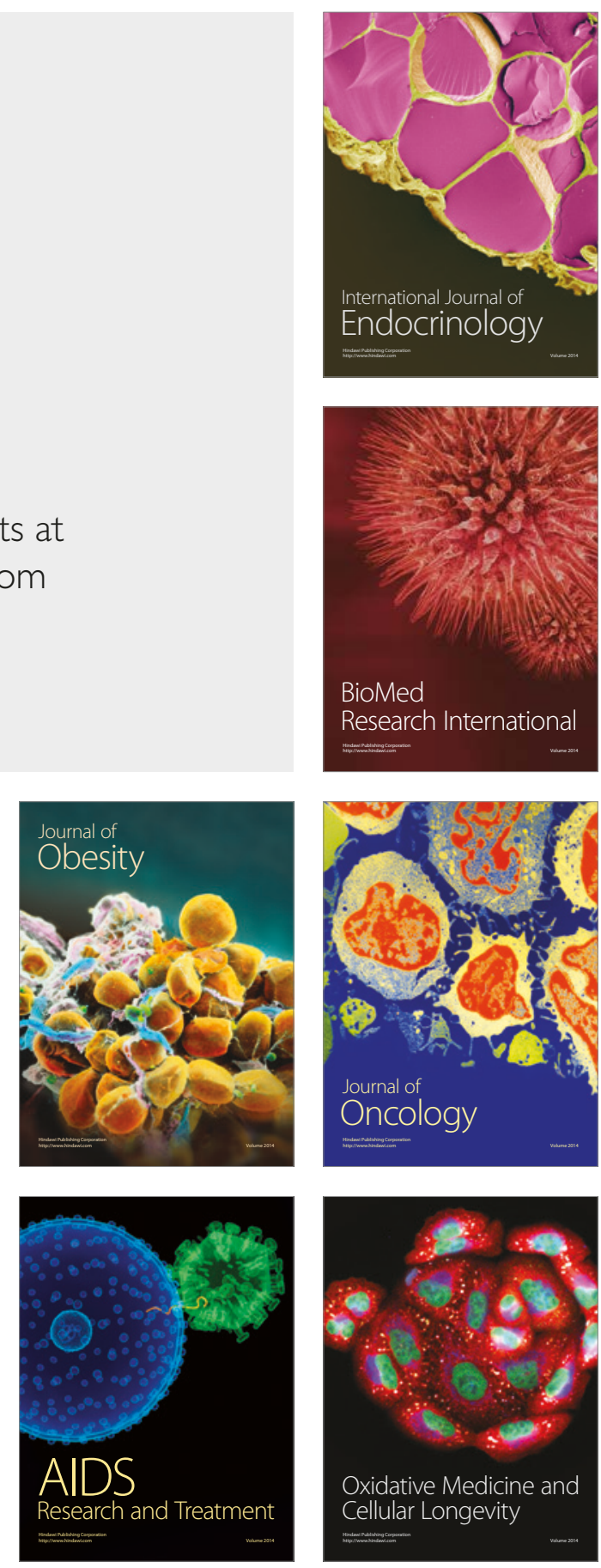